\title{
On Structural Analyses of the ITER Vacuum Vessel Bolometer Camera Housing Conceptual Design
}

\author{
N. Jaksic ${ }^{\mathrm{a}}$, H. Meister ${ }^{\mathrm{a}}$, F. Penzel ${ }^{\mathrm{a}}$, D. Hermann ${ }^{\mathrm{a}}$, A. Pataki ${ }^{\mathrm{a}}$, R. Reichle ${ }^{\mathrm{b}}$ \\ ${ }^{a}$ Max-Planck-Institut für Plasmaphysik, Boltzmannstr. 2, D-85748 Garching, Germany \\ bITER Organisation, Route de Vinon-sur-Verdon, CS 90 046, 13067 St. Paul Lez Durance Cedex, France
}

\begin{abstract}
The ITER bolometer provides an absolutely calibrated measurement of the radiation emitted by the plasma, which is a part of the total energy balance. The development is especially challenging because of the extreme environmental conditions within the vacuum vessel (VV) during plasma operation. The bolometer has to guarantee reliable measurements within an environment characterized by high neutron flux as well as temperatures exceeding $200^{\circ} \mathrm{C}$. In addition to the thermal loads, the bolometer body is exposed to the mechanical loads caused by electromagnetic forces during transient events of plasma disruption. This paper describes a possible procedure for a structural analysis of the bolometer camera body. To examine all-important structural properties of the bolometer body, a multiple nonlinear finite element model based on a CAD conceptual design has been generated. Subsequently, a transient mechanical analysis has been performed using the finite element code ANSYS. A general electromagnetic model, taking into account the contribution of all structural parts and electromagnetic loading starting with the DINA code, generated the input for the analysis. From the wide range of DINA results, the worst-case load scenario has been chosen. The results of the structural analysis regarding the camera housing structural integrity are presented and discussed here.
\end{abstract}

Keywords: Bolometer, ITER, diagnostics, finite element analysis

\section{Introduction}

The ITER bolometer provides an absolutely calibrated measurement of the radiation emitted by the plasma, which is a part of the total energy balance. The bolometer cameras are located at the walls of the structure facing the plasma. Considered in detail, the cameras will be installed in equatorial port 1 , in the upper ports 1 and 17 , in 5 divertor cassettes $(18,26,44,49$ and 54) and behind blanket modules (BM) in the vacuum vessel sectors: 1,3 , 4, 6, 8 and 9 [1]. Overall, 71 cameras are delivering 550 lines of sight (LOS). The camera structure development is especially challenging because of the extreme environmental conditions within the vacuum vessel (VV) during plasma operation. The bolometer has to guarantee reliable measurements within an environment characterized by high neutron flux as well as temperatures exceeding $200{ }^{\circ} \mathrm{C}$. In addition to the thermal loads, the bolometer body is exposed to the mechanical loads caused by electromagnetic forces during plasma disruptions. Due to the available space envelopes and integration constraints within the plasma chamber the camera, structures will have different designs varying with location.

This paper describes a structural analysis of the bolometer camera housing loaded by electromagnetic (EM) forces induced due to plasma disruption, which is proposed to be installed in the VV sectors 1 behind BM 1.

\section{VV bolometer camera design}

The CAD model of the VV bolometer camera housing conceptual design, used in structural analysis including only current conducting parts, is shown in Fig. 1. The interior of the bolometer camera, mainly comprising the printed circuit board (PCB) made of ceramic carrying the sensor, is hidden in the picture.

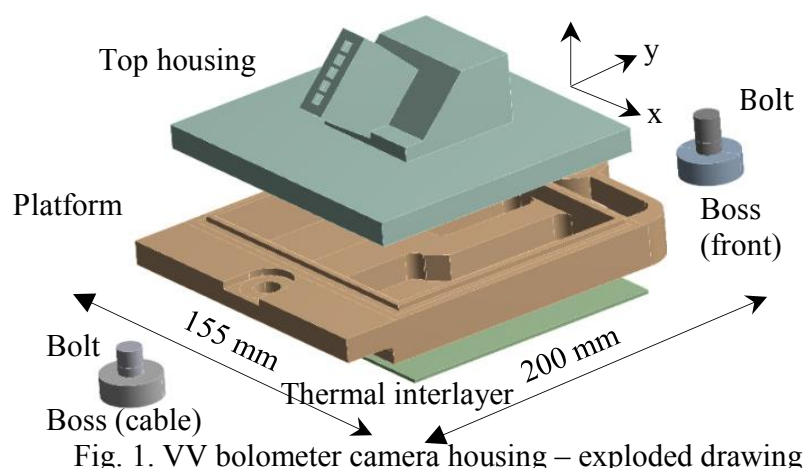

The camera housing is attached by means of two bosses welded on the VV inner surface and screwed with two M12 bolts. An interlayer between the camera platform and the VV has to ensure a good thermal contact. More details on the design are given in [1].

\section{Analysis description}

The structural analysis described here is only a part of integrated multiphysics numerical analyses. It is mainly based on an EM analysis [2], which is performed in a socalled $20^{\circ}$ general cyclic-symmetric model representing for EM analysis all relevant structural parts. The evolution of magnetic fields and plasma current used as input were taken from results of the DINA code [3].

The model of the camera housing was embedded into $20^{\circ}$ general cyclic-symmetric model environment and the mesh of the camera housing was adapted to the general model mesh. Accordingly, it is quite rough. The present structural analysis is performed with the actual camera housing design, which comprises all geometrical details important for such an analysis. The distribution of the cameras within the $\mathrm{VV}$ behind the blanket module in sectors 1 is shown in Fig. 2a with the zoom at the position 


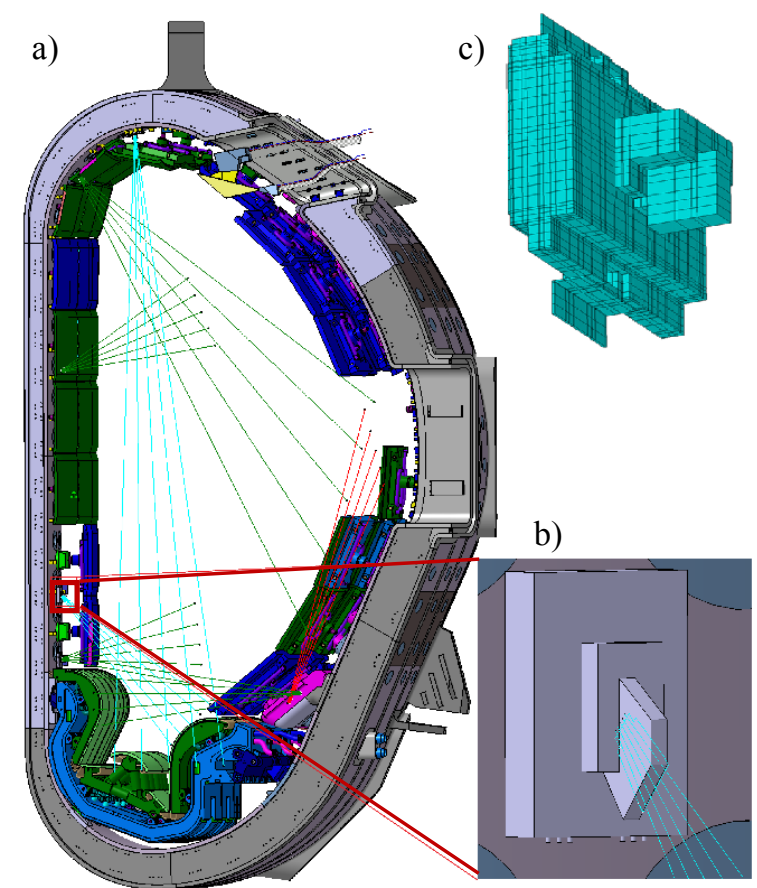

Fig. 2. Bolometer distribution within VV at the sector 1

of the analysed bolometer b) and c) the simplification by meshing within the general EM model.

The reason for an EM analysis of the camera housing within the so-called general $20^{\circ} \mathrm{EM}$ model is that the surrounding structural parts within the $\mathrm{VV}$ provide the electromagnetic shielding of the camera housing. A simple analysis of the camera housing neglecting this shielding would deliver EM forces and moments at an order of magnitude larger.

Accordingly, the general $20^{\circ}$ EM model consists of following items: the $\mathrm{VV}$ with the inner and outer parts including the ribs, ports (lower, equatorial and upper), divertor structures and the blanket modules. Moreover, for preferably precise results, the interior (vacuum) as well as the outer space (air) up to the diameter of $80 \mathrm{~m}$ has been modelled. The ANSYS 19 (classic) code has been used for analysis, performed by means of a 3D solid model. The so-called SOLID 97 element type enables the use of vector potential formulation with 4 degrees of freedom (DOF): $A_{x}, A_{y}, A_{z}$ and the time-integrated electric potential Volt. Additionally, the so-called INFIN 111 element type has been defined on the free (air) surfaces simulating the far-field decay effect in magnetic field.

The general $20^{\circ} \mathrm{EM}$ model has to be prepared in a special way to be able to use the output of the DINA code as boundary conditions. According to the previous analyses, the most loaded bolometer position is marked in Fig. 2 with the red square. The worst loading case of the plasma transient for bolometer housing at this position is the so-called vertical displacement event (VDE) with the features denoted as "Down (DW) Fast lin36ms Cat. III". The resulting eddy current contribution is considered in the present analysis only. It can be shown [4] that the Halo-Current contribution is negligible.
Two different EM analyses for both the toroidal and the poloidal field variation have been performed and the results have been added in the post-processing phase. The toroidal field is not included in the analysis model explicitly, because its intensity and direction does not change during the disruption. Corresponding procedures can be carried out during the post processing knowing that inside the $\mathrm{VV}$ the static toroidal field is given with: $B_{t}=$ $5.3 \cdot 6.2 / r$, where $r$ is the radial distance from the plasma center (minor plasma radius).

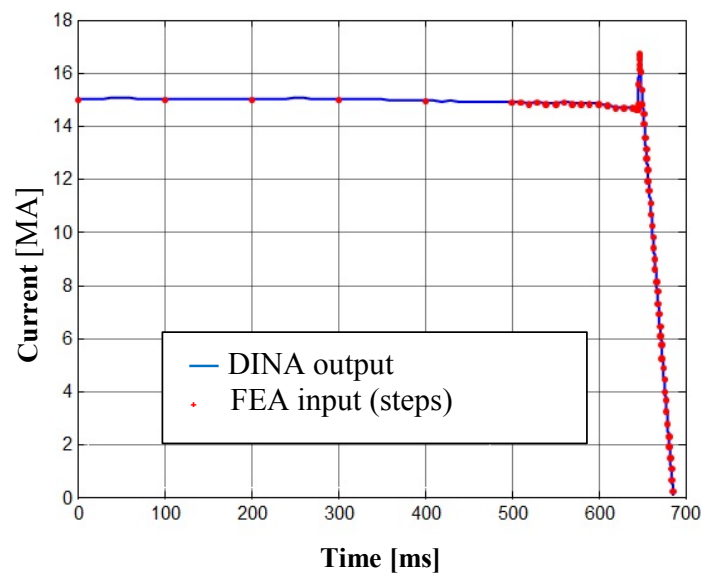

Fig. 3. Total plasma current VDE DW fast lin36ms Cat. III

While the DINA code uses a very fine time increment with 492 time steps, for the FE analysis only 90 points in time have been chosen. Knowing that ANSYS applies a linear interpolation between two points, the steps distribution in time have been adapted adequately, like shown in Fig.3.

\section{FE local model description}

While the bolometer housing geometry within the global model (Fig. 2b) is in highly simplified terms and roughly meshed (Fig. 2c) only, the local model based on the real design including all significant geometrical features is meshed with a very fine grid (Fig. 4.).

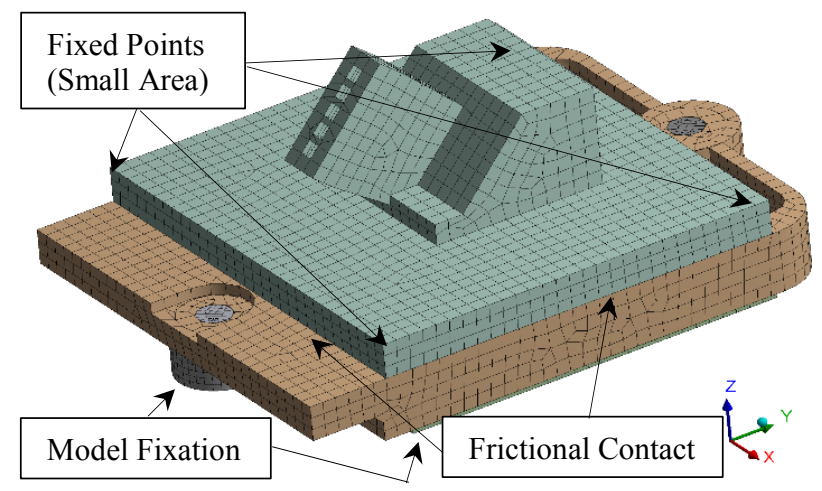

Fig. 4. FE local model of the bolometer camera housing

The entire local model structure is meshed with linear hexahedral elements to be compatible with the general EM model mesh. The model is geometrically nonlinear, consisting of top housing, platform, thermal interlayer, two bosses and two bolts. Between all single parts, contact surfaces with a coefficient of friction of 0.2 are defined. To simulate the fixation of the platform to the VV wall, a so-called fixed support is defined at the welded region of the bosses, i.e. all DOF are removed. The free surface of 
the thermal interlayer is statically determinate and additionally held by so-called compression only support. The pretension force at the bolts is defined with $5000 \mathrm{~N}$. The EM nodal force determined by the general model was transferred to the local model without additional examination. Due to the fact that the general EM model and the structural local model do not only have different coordinate systems, but also different meshes and even slightly different geometry, special attention has been paid to the translation of the EM nodal forces. Moreover, while the general EM model was developed for use with ANSYS classic, ANSYS Workbench [5] was used for local model analysis. The original EM loads have been stored for each time step (90 in total) and each individual structural part (5 parts in total) resulting in 450 files. Several consecutive interfaces and macros have been written for data transfer including the use of the ANSYS intrinsic function for searching the nearest node to a coordinate. Fig. 5 shows the nodal EM forces and resulting effects at a certain point of time in two planes, front view a) and top view b).

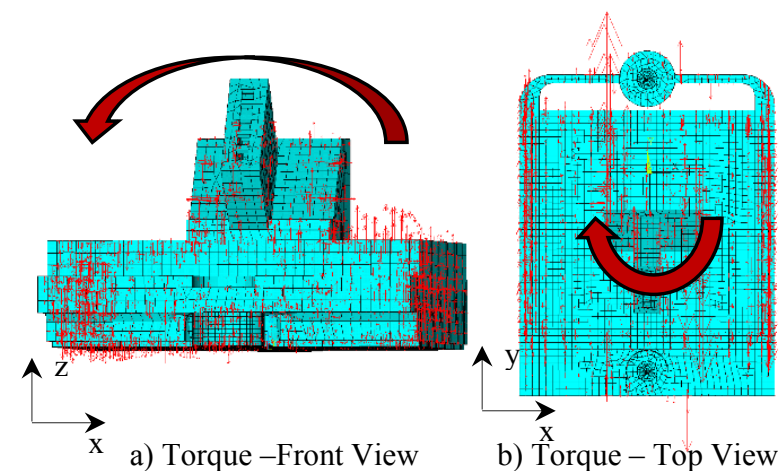

Fig. 5. Nodal EM forces and resulting effects

\section{Results and discussion}

A transient dynamic analysis has been performed to analyse the structural response, excited by time varying EM loads, which were applied as the nodal forces. The main aims of the analysis were to assess the general structural behaviour, as well as to support the choice of material.

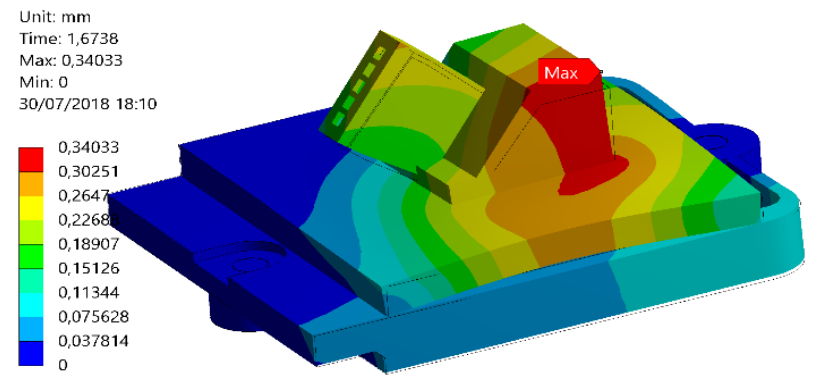

Fig. 6. Displacements at $0.6738 \mathrm{sec}$. of the transient analysis

Two materials have been proposed, TZM and $\mathrm{CuCr}(1) \mathrm{Zr}$. Due to the superior thermal conductivity $\mathrm{CuCr}(1) \mathrm{Zr}$ was chosen and used for the structural assessment. It has a Young's modulus of $120 \mathrm{GPa}$ and a Poisson ratio of 0.33 .

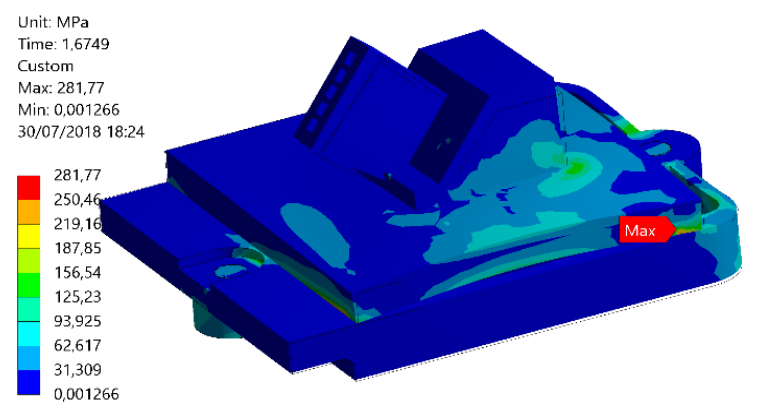

Fig. 7. Mises Stress at $0.675 \mathrm{sec}$. of the transient analysis

The bosses and the thermal interlayer are made of stainless steel and $\mathrm{Cu}$ respectively. 90 non-uniform time steps (Fig. 3) with an interval of $0.1-0.688$ s. have been chosen for the analysis. The comprehensive result plots for e.g. stresses and displacements cannot be shown by a single illustration, because the results are a function of time. Some snapshots with maximal values as examples are shown in Figs. 6 and 7. The peak stresses are travelling through the structure changing magnitude and region for each time step. The maximum Mises stress on the top housing is $282 \mathrm{MPa}$ at $0.675 \mathrm{~s}$. of the transient analysis and $320 \mathrm{MPa}$ on the platform at $0.647 \mathrm{~s}$. Accordingly, the reaction forces at the bosses are changing value and direction during the plasma transient events as well. Fig. 8 shows a snapshot of the reaction force on the cable side boss, at the moment when the maximum value is reached. This is a tensile force for the boss structure.

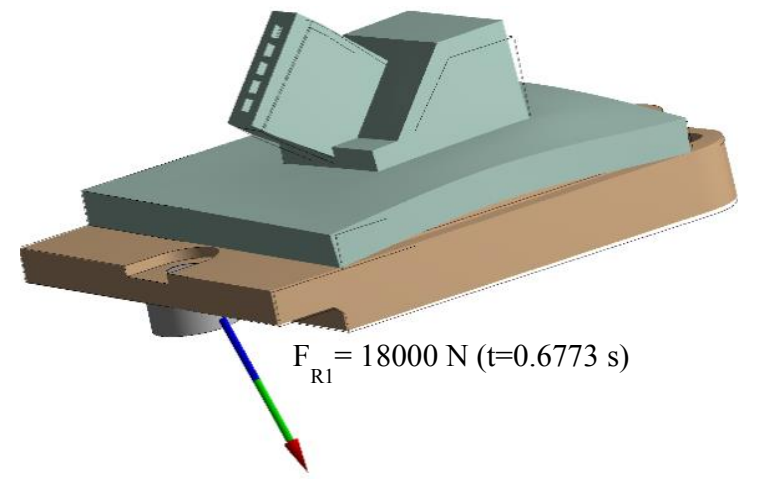

Fig. 8. Reaction force at the cable boss $(0.667 \mathrm{sec}$.)

The entire set of results for the reaction forces and moments at both bosses are stored in files for all mainand sub-steps resulting in 864 time points.

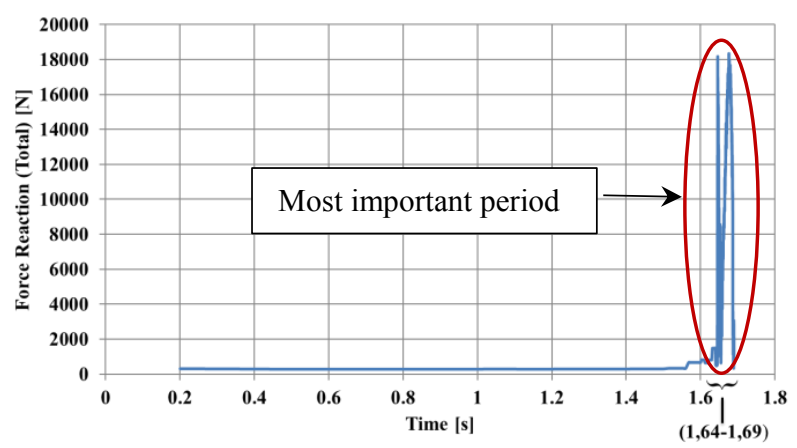

Fig. 9. Force reaction over time on the cable boss 


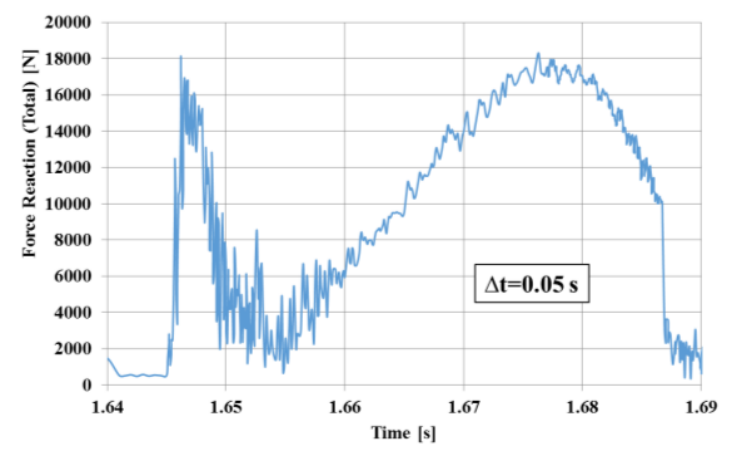

Fig. 10. Zoom in Fig. 9. from $1.64-1.69 \mathrm{sec}$.

Note, that the structural FE analysis has a different total analysis time length than the EM analysis. The time dependent response for the reaction force on the cable boss is shown in the Fig. 9. Zooming into the most important period of this diagram, displays a force reaction time response within $0.05 \mathrm{~s}$ (Fig.10). The Mises peak stress on the cable boss at the so-called critical crosssection at $1.6763 \mathrm{~s}$. is shown in Fig. 11. The line in the figure marked with $\overline{A B}$ is the so-called stress classification line (SCL) used in fatigue analysis.

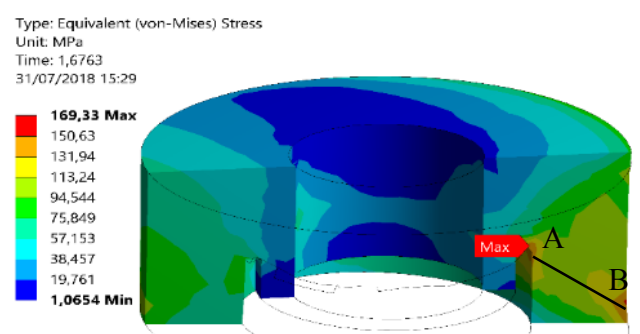

Fig. 11. Mises stress on critical cross-section, boss cable side

Related to the allowable stresses in $\mathrm{CuCr}(1) \mathrm{Zr}$ reliable data for the material batch used is required. It is proposed to use $\mathrm{CuCr}(1) \mathrm{Zr}$ delivered in so-called solution-annealed and aged condition (SAA), (Fig. 12), with material parameters as defined in [6]. It can therefore be concluded that the average yield stress $\left(\sigma_{y}\right)$ is $\sim 200 \mathrm{MPa}$. A simplified tensile design stress $\left(\sigma_{\text {design }}\right)$ can be defined as the minimum value of $2 / 3$ of the yield strength. Accordingly, for the given material example the design value for stress should be $\sigma_{\text {design }} \sim 150 \mathrm{MPa}$.

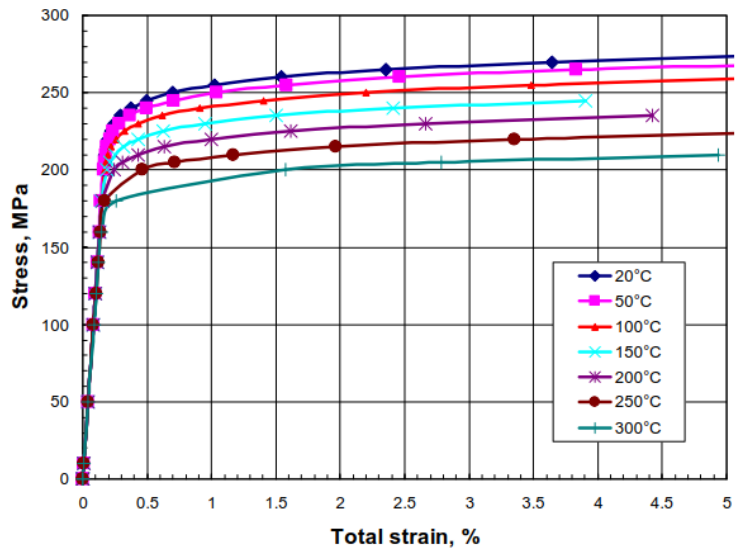

Fig. 12. Stress-strain diagram for $\mathrm{CuCrZr}$ (SAA) [6]

\section{Conclusions}

This paper presents a possible procedure for a structural analysis of the VV bolometer camera housing. The described analysis is a part of a complex numerical analysis which has to be carried out in several stages. The complexity of the analysis lies in the plasma disruption phenomena, which implies a staged approach based on numerical multiphysics analysis. The output of the DINA code was used as input for an EM analysis, whose results was used in a transient structural analysis using ANSYS. Beside the work needed for generation of different FE models used in different codes and solution modules, a significant effort is required for establishing the interfaces needed for the data transfer within certain solution steps. The main objectives of the present analysis are the general structural assessment and the choice of material. Based on the superior thermal conductivity $\mathrm{CuCr}(1) \mathrm{Zr}$ was chosen for the camera housing. The maximum Mises stress at the camera housing structure found was $320 \mathrm{MPa}$. Considering the stress design value of $150 \mathrm{MPa}$ according to the example given in this paper, the stress values gained by the present analyses exceeded this limit. The fact, that these peak stresses are limited to a very small region, a reduction can be reached by modifications of the housing design. The maximum Mises peak stress on the bosses amounts to $\sim 170 \mathrm{MPa}$, which is in any case under the stress design limit for stainless steel. Nevertheless, a fatigue analysis of the bosses is required, because the bosses are attached to the $\mathrm{VV}$, which is part of the nuclear confinement barrier and thus a critical element to validate.

\section{Acknowledgments}

This work was partly supported by Fusion for Energy under the Grant F4E-FPA-384-SG03. The views expressed in this publication are the sole responsibility of the authors and do not necessarily reflect the views of Fusion for Energy and the ITER Organization. Neither Fusion for Energy nor any person acting on behalf of Fusion for Energy is responsible for the use, which might be made of the information in this publication. The authors would like to thank the company LT Calcoli to make available the ITER general EM model. Especially, thanks go to José Manuel Sanchez, Anna Marin and Irene Pagani for their help in realising this paper.

\section{References}

[1] A. Pataki, et al., Flexible VV bolometer camera design in ITER to adapt to the final position of the gaps between Blanket Modules, (2018) 30 $30^{\text {th }}$ SOFT.

[2] R. Roccella, et al., Procedures to interface plasma disruption simulations and finite element electromagnetic analyses, 24 ${ }^{\text {th }}$ IAEA, (2012) 285.

[3] R.R. Khayrutdinov, et al., Studies of plasma equilibrium and transport in a tokamak fusion device with the inversevariable technique, J. Comp. Phys. 109 (1993) 193-201.

[4] M. Roccella, et al., Detailed electromagnetic numerical evaluation of eddy currents induced by toroidal and poloidal magnetic field variation and halo currents, Fusion Engineering and Design 83, (2008) 1625-1630.

[5] ANSYS, Inc. Products Release 19.0, (2018).

[6] ITER Organisation, Material Properties Handbook, No. G 74 MA 9 00-11-10 W 0.1, (2001). 